\title{
ROUTING NUMBERS OF CYCLES, COMPLETE BIPARTITE GRAPHS, AND HYPERCUBES*
}

\author{
WEI-TIAN LI ${ }^{\dagger}$, LINYUAN LU ${ }^{\dagger}$, AND YITING YANG ${ }^{\dagger}$
}

\begin{abstract}
The routing number $\operatorname{rt}(G)$ of a connected graph $G$ is the minimum integer $r$ so that every permutation of vertices can be routed in $r$ steps by swapping the ends of disjoint edges. In this paper, we study the routing numbers of cycles, complete bipartite graphs, and hypercubes. We prove that $r t\left(C_{n}\right)=n-1$ (for $n \geq 3$ ) and for $s \geq t, r t\left(K_{s, t}\right)=\left\lfloor\frac{3 s}{2 t}\right\rfloor+O(1)$. We also prove $n+1 \leq r t\left(Q_{n}\right) \leq 2 n-2$ for $n \geq 3$. The lower bound $r t\left(Q_{n}\right) \geq n+1$ was previously conjectured by Alon, Chung, and Graham [SIAM J. Discrete Math., 7 (1994), pp. 513-530]. A variation, called fractional routing number, is also considered in this paper.
\end{abstract}

Key words. routing number, parallel sorting algorithm

AMS subject classifications. 05C, 68

DOI. $10.1137 / 090776317$

1. Introduction. The problem of routing permutations over graphs arose in different fields $[2,3,6,7]$, such as the study of communicating processes on networks, the data flow on parallel computation, and the analysis of routing algorithms on VLSI chips. This problem can be described as follows: Let $G=(V, E)$ be a connected graph with vertices $\left\{v_{1}, v_{2} \ldots v_{n}\right\}$ and $\pi$ be a permutation on $[n]$. Initially, each vertex $v_{i}$ of $G$ is occupied by a "pebble." The pebble on $v_{i}$ will be labeled as $p_{j}$ if $\pi(i)=j$. Pebbles can be moved around by the following rule. At each step a disjoint collection of edges of $G$ is selected, and the pebbles at each edge's two endpoints are interchanged. The goal is to move/route each pebble $p_{i}$ to its destination $v_{i}$. Define $\operatorname{rt}(G, \pi)$ to be the minimum number of steps to route the permutation $\pi$. Finally, define $r t(G)$ the routing number of $G$ by

$$
r t(G)=\max _{\pi} \operatorname{rt}(G, \pi) .
$$

Determining the routing number of a graph is a quite difficult problem. For a few kinds of special graphs, the routing numbers are known in the literature [4]. For the path $P_{n}(n \geq 3), r t\left(P_{n}\right)=n$; for the complete graph $K_{n}(n \geq 3), r t\left(K_{n}\right)=2$; for the complete bipartite graph $K_{n, n}(n \geq 3), r t\left(K_{n, n}\right)=4$; and for the star $S_{n}(n \geq 3)$, $r t\left(S_{n}\right)=\left\lfloor\frac{3(n-1)}{2}\right\rfloor$.

Except for those exact results, some upper/lower bounds were obtained for certain graphs. A trivial lower bound is

$$
r t(G) \geq D(G) .
$$

Here $D(G)$ is the diameter of $G$. If $G$ has a connected spanning subgraph $H$, then

$$
r t(G) \leq r t(H) .
$$

${ }^{*}$ Received by the editors November 6, 2009; accepted for publication (in revised form) September 10, 2010; published electronically November 4, 2010.

http://www.siam.org/journals/sidma/24-4/77631.html

†Department of Mathematics, University of South Carolina, Columbia, SC 29208 (li37@mailbox. sc.edu, lu@math.sc.edu, yang36@mailbox.sc.edu). The second and third authors were supported in part by NSF grant DMS 0701111. 
In particular, we have

$$
r t(G) \leq r t\left(T_{n}\right)
$$

where $T_{n}$ is a spanning tree of $G$. Alon, Chung, and Graham [1] proved

$$
r t\left(T_{n}\right) \leq 2 n,
$$

and they conjectured that

$$
r t\left(T_{n}\right) \leq\left\lfloor\frac{3(n-1)}{2}\right\rfloor
$$

and equality holds if and only if $T_{n}$ is a star or $P_{4}$. This conjecture is still open. However, Zhang [8] proved that it is asymptotically correct by showing

$$
r t\left(T_{n}\right) \leq\left\lfloor\frac{3 n}{2}\right\rfloor+O(\log n) .
$$

Alon, Chung, and Graham gave an upper bound for the routing number of complete bipartite graph $K_{s, t}, s \geq t$,

$$
r t\left(K_{s, t}\right) \leq 2\left\lceil\frac{s}{t}\right\rceil+2 .
$$

An important class of graphs is the hypercube $Q_{n}$, which is one of the most popular topological models in parallel computing. The previously best known bound for $r t\left(Q_{n}\right)$ is

$$
n \leq r t\left(Q_{n}\right) \leq 2 n-1 .
$$

The lower bound comes from $r t\left(Q_{n}\right) \geq D\left(Q_{n}\right)=n$. The upper bound is a consequence of the following result on the Cartesian product of two graphs [1].

$$
r t\left(G_{1} \square G_{2}\right) \leq 2 r t\left(G_{1}\right)+r t\left(G_{2}\right) .
$$

Alon, Chung, and Graham [1] conjectured $\operatorname{rt}\left(Q_{n}\right) \geq n+1$ for $n \geq 2$. Here we settle this conjecture by showing the following theorem.

TheOREM 1.1. Let $Q_{n}$ be the $n$-cube, then for $n \geq 3$

$$
n+1 \leq r t\left(Q_{n}\right) \leq 2 n-2 .
$$

The improvements of both upper bound and lower bound are very limited. Alon, Chung, and Graham have a stronger conjecture $\operatorname{rt}\left(Q_{n}\right)=n+O(1)$ [1]. Our result is just the first step toward this conjecture.

Furthermore, we have the following result on the cycle $C_{n}$.

TheOrem 1.2. Let $C_{n}$ be a cycle of length $n$, then

$$
r t\left(C_{n}\right)=n-1 \text {. }
$$

We have the following theorem for the routing number of the complete bipartite graph $K_{s, t}$ with $s \geq t$.

TheOREM 1.3. For the complete bipartite graph $K_{s, t}$ with $s \geq t \geq 1$,

$$
\left\lfloor\frac{3 s}{2 t}\right\rfloor-1 \leq r t\left(K_{s, t}\right) \leq\left\lfloor\frac{3 s}{2 t}\right\rfloor+7 .
$$

Copyright (c) by SIAM. Unauthorized reproduction of this article is prohibited. 
In other words, $r t\left(K_{s, t}\right)=\left\lfloor\frac{3 s}{2 t}\right\rfloor+O(1)$.

We will prove several lemmas on the lower bounds for the routing numbers of some graphs in section 2. The proofs of theorems are in section 3. A variation, called fractional routing number, is given in section 4. It deepens our understanding of various lower bounds for the routing numbers. In the last section, we conclude the paper by posing some problems.

2. Lower bounds for routing numbers. In this section, we will study lower bounds for routing numbers. Let $C_{n}$ be a cycle on $n$ vertices. The vertices are labeled as $v_{1}, v_{2} \ldots, v_{n}$ in the cyclic order so that the edges are $e_{i}=v_{i} v_{i+1}$ for $i=1, \ldots, n-1$ and $e_{n}=v_{n} v_{1}$.

Lemma 2.1. For the cycle $C_{n}$ on $n$ vertices, we have

$$
r t\left(C_{n}\right) \geq n-1 .
$$

Proof. Let $\rho$ be the cyclic rotation $(123 \cdots n)$ on $[n]$. We claim $r t\left(C_{n}, \rho\right) \geq n-1$.

Otherwise, suppose we can route $\rho$ in at most $n-2$ steps. Let $R$ be such a routing process. We say a pebble has a forward move if it is on $v_{i}$ and moved to $v_{i+1}$, or it is on $v_{n}$ and moved to $v_{1}$. By a backward move, we mean that a pebble is moved from $v_{i+1}$ to $v_{i}$, or from $v_{1}$ to $v_{n}$. For $1 \leq i \leq n$, define $R^{+}\left(p_{i}\right)$ to be the number of forward moves of $p_{i}$ and $R^{-}\left(p_{i}\right)$ to be the number of backward moves of $p_{i}$. For $1 \leq i \leq n$, we have

$$
R^{+}\left(p_{i}\right)-R^{-}\left(p_{i}\right) \equiv 1 \bmod n .
$$

By assumption, this routing process takes at most $n-2$ moves. We have

$$
\begin{gathered}
0 \leq R^{+}\left(p_{i}\right) \leq n-2, \\
0 \leq R^{-}\left(p_{i}\right) \leq n-2, \\
-(n-2) \leq R^{+}\left(p_{i}\right)-R^{-}\left(p_{i}\right) \leq n-2 .
\end{gathered}
$$

Equations (2.1) and (2.2) together imply for $1 \leq i \leq n$,

$$
R^{+}\left(p_{i}\right)-R^{-}\left(p_{i}\right)=1 .
$$

Thus $\sum_{i=1}^{n}\left(R^{+}\left(p_{i}\right)-R^{-}\left(p_{i}\right)\right)=n$.

On the other hand, each swap is a forward move of one pebble but also a backward move of another pebble, so we have

$$
\sum_{i=1}^{n}\left(R^{+}\left(p_{i}\right)-R^{-}\left(p_{i}\right)\right)=0 .
$$

Contradiction.

This idea can be generalized to obtain a lower bound for the routing number of the Cartesian product of two graphs. For two graphs $G=(V, E)$ and $G^{\prime}=\left(V^{\prime}, E^{\prime}\right)$, the Cartesian product $G \square G^{\prime}$ is the graph with vertex set $V \square V^{\prime}=\left\{\left(v, v^{\prime}\right) \mid v \in V, v^{\prime} \in V^{\prime}\right\}$ and with $\left(u, u^{\prime}\right)\left(v, v^{\prime}\right)$ as an edge of $G \square G^{\prime}$ if and only if either $u=v, u^{\prime} v^{\prime} \in E^{\prime}$ or $u^{\prime}=v^{\prime}, u v \in E$. A graph $G$ is called an $m$-routing graph if there is a permutation $\sigma$ such that the distance between each pebble $p_{i}$ and its destination $v_{i}$ is at least $m$.

Copyright (c) by SIAM. Unauthorized reproduction of this article is prohibited. 
Lemma 2.2. Let $G$ be an m-routing graph, then

$$
r t\left(C_{n} \square G\right) \geq n+m-1 .
$$

Proof. We can picture $C_{n} \square G$ as an array with each row spanning a copy of $C_{n}$ and each column spanning a copy of $G$. Let $\pi$ be the desired routing permutation such that each pebble at vertex $(c, g)$ has the destination $(\rho(c), \sigma(g))$, where $\rho=(123 \cdots n)$ is the cyclic rotation of $C_{n}$ and $\sigma$ is the permutation of $G$ in the definition of $m$-routing graph.

Just as in the proof of Lemma 2.1, there is a pebble moved at least $n-1$ steps in horizontally. From $d(f, \sigma(f)) \geq m$, we need at least $m$ vertical steps to route it. So totally we need $n+m-1$ steps.

\section{Proof of theorems.}

Proof of Theorem 1.1. Note that $Q_{n}=C_{4} \square Q_{n-2}$ and $Q_{n-2}$ is an $n$-2-routing graph. By Lemma 2.2, we have

$$
r t\left(Q_{n}\right) \geq 4+(n-2)-1=n+1 .
$$

The upper bound is due to a computational result (see the concluding remarks of the last section)

$$
r t\left(Q_{3}\right)=4 .
$$

This is done by an exhausting computer search. Applying (1.1), we have

$$
\begin{aligned}
r t\left(Q_{n}\right) & \leq r t\left(Q_{n-1}\right)+2 \\
& \leq \cdots \\
& \leq r t\left(Q_{3}\right)+2(n-3) \\
& =4+2(n-3) \\
& =2 n-2 . \quad
\end{aligned}
$$

The following algorithm is essentially the same as the odd-even transposition sort in parallel sorting networks (see [5] for a comprehensive survey). We rephrase it with our terminologies.

Denote the vertices of $P_{n}$ by $v_{1}, \ldots, v_{n}$ in order and each edge $v_{i} v_{i+1}$ by $e_{i}$ for $i=1, \ldots, n-1$. We call $e_{i}$ an odd edge if $i$ is odd, and an even edge provided $i$ is even. We interchange two pebbles on edge $e_{i}$ if the pebble on $v_{i}$ has an index greater than that of the pebble on $v_{i+1}$. In odd steps, we only exchange the pebbles on odd edges, while in even steps we exchange the pebbles on even edges. We exchange the pebbles on odd edges and even edges in every other steps.

This method routes any permutation of pebbles on $P_{n}$ in at most $n$ steps.

However, it is natural to ask, when do we need $n$ steps to route a permutation of pebbles on $P_{n}$ ? Can it be done by less steps? The following lemma gives a sufficient condition of routing a permutation on $P_{n}$ in at most $n-1$ steps.

Lemma 3.1. Given a permutation $\pi$ on $[n]$, if $p_{1}$ is on $v_{i}$ and $p_{n}$ is on $v_{j}$ for some $i<j$, then $r t\left(P_{n}, \pi\right) \leq n-1$.

Proof. Let $S_{i}=p_{i_{1}} p_{i_{2}} \cdots p_{i_{n}}$ be the list of pebbles on $v_{1}, \ldots, v_{n}$ after we finished step $i$, where $S_{0}$ is just the initial distribution of pebbles when $\pi$ is given. There will be $k+1$ lists if we route $\pi$ in $k$ steps. For each $S_{i}, i \geq 1$, if $p_{1}$ is on $v_{\ell}$ and $p_{n}$ is on 
$v_{m}$, define $T_{i-1}$ to be the following list.

$$
T_{i-1}=\underbrace{p_{(i-1)_{1}} \cdots p_{(i-1)_{\ell-1}}}_{\text {from } S_{i-1}} \underbrace{p_{i_{\ell+1}} \cdots p_{i_{m-1}}}_{\text {from } S_{i}} \underbrace{p_{(i-1)_{m+1}} \cdots p_{(i-1)_{n}}}_{\text {from } S_{i-1}} \text { for } i=1, \ldots, k \text {. }
$$

In other words, we concatenate the first $\ell-1$ pebbles and the last $n-m$ pebbles in $S_{i-1}$ together to the middle pebbles $p_{i_{\ell+1}} \cdots p_{i_{m-1}}$ in $S_{i}$, forming a new list of pebbles.

For example, consider the permutation

$$
\pi=\left(\begin{array}{ccccccc}
1 & 2 & 3 & 4 & 5 & 6 & 7 \\
4 & 5 & 1 & 2 & 7 & 3 & 6
\end{array}\right)
$$

\begin{tabular}{|c|c|c|c|c|c|c|c|c|c|c|c|c|}
\hline$p_{4}$ & $p_{5}$ & $p_{1}$ & $p_{2}$ & $p_{7}$ & $p_{3}$ & $p_{6}$ & \multirow[b]{2}{*}{$T_{0}$} & \multirow[b]{2}{*}{$p_{4}$} & \multirow[b]{2}{*}{$p_{5}$} & \multirow[b]{2}{*}{$p_{2}$} & \multirow[b]{2}{*}{$p_{3}$} & \multirow[b]{2}{*}{$p_{6}$} \\
\hline & & & & & & & & & & & & \\
\hline$p_{4}$ & $p_{5}$ & $p_{1}$ & $p_{2}$ & $p_{3}$ & $p_{7}$ & $p_{6}$ & \multirow[b]{2}{*}{$T_{1}$} & & & & & \\
\hline & & & & & & & & $p_{4}$ & $p_{5}$ & $p_{2}$ & $p_{3}$ & $p_{6}$ \\
\hline$p_{4}$ & $p_{1}$ & $p_{5}$ & $p_{2}$ & $p_{3}$ & $p_{6}$ & $p_{7}$ & \multirow[b]{2}{*}{$T_{2}$} & & & & & \\
\hline & & & & & & & & $p_{4}$ & $p_{2}$ & $p_{5}$ & $p_{3}$ & $p_{6}$ \\
\hline$p_{1}$ & $p_{4}$ & $p_{2}$ & $p_{5}$ & $p_{3}$ & $p_{6}$ & $p_{7}$ & \multirow[b]{2}{*}{$T_{3}$} & & & & & \\
\hline & & & & & & & & $p_{2}$ & $p_{4}$ & $p_{3}$ & $p_{5}$ & $p_{6}$ \\
\hline$p_{1}$ & $p_{2}$ & $p_{4}$ & $p_{3}$ & $p_{5}$ & $p_{6}$ & $p_{7}$ & \multirow[b]{2}{*}{$T_{4}$} & & & & & \\
\hline & & & & & & & & $p_{2}$ & $p_{3}$ & $p_{4}$ & $p_{5}$ & $p_{6}$ \\
\hline$p_{1}$ & $p_{2}$ & $p_{3}$ & $p_{4}$ & $p_{5}$ & $p_{6}$ & $p_{7}$ & & & & & & \\
\hline
\end{tabular}

Note that $p_{1}$ is on $v_{3}$ and $p_{7}$ is on $v_{5} . T_{i}$ 's are shown in the following diagram.

Now we claim that

(1) Each $T_{i}$ contains exactly the $n-2$ pebbles $p_{2}, p_{3}, \ldots, p_{n-1}$.

(2) The lists $T_{0}, T_{1}, \ldots, T_{k-1}$ are corresponding to the process of routing $n-2$ pebbles on the path $P_{n-2}$ using the odd-even transposition.

The following arguments we discuss about $p_{1}$ all work for $p_{n}$ as well.

To show (1), notice that in the list $S_{i}$, the set $\left\{p_{i_{1}}, \ldots, p_{i_{n}}\right\} \backslash\left\{p_{i_{\ell}}, p_{i_{m}}\right\}$ contains exactly the pebbles $p_{2}, \ldots, p_{n-1}$ by our assumption. Since $p_{1}$ is placed on $v_{\ell}$ in $S_{i}$, at step $i$ either we swap $p_{1}$ on $v_{\ell+1}$ and some pebble $v_{\ell}$, or $p_{1}$ is already on $v_{\ell}$ and we do not swap it with the pebble on $v_{\ell+1}$. If a pebble is on $v_{j}$ for some $j \leq \ell$, it cannot "jump" over $v_{\ell}$ at step $i$. Thus, $\left\{p_{i_{1}}, \ldots, p_{i_{\ell-1}}\right\}$ should be the same as $\left\{p_{(i-1)_{1}}, \ldots, p_{(i-1)_{\ell-1}}\right\}$. Hence $T_{i}$ contains exactly the $n-2$ pebbles $p_{2}, p_{3}, \ldots, p_{n-1}$.

For (2), if at step $i p_{1}$ is moved from $v_{\ell+1}$ to $v_{\ell}$, it means we are exchanging pebbles on edges $v_{\ell-2} v_{\ell-1}, v_{\ell-4} v_{\ell-3}, \ldots$ in $S_{i-1}$, which are $p_{(i-1)_{\ell-2}} p_{(i-1)_{\ell-1}}$, $p_{(i-1)_{\ell-4}} p_{(i-1)_{\ell-3}}, \ldots$ Thus, at step $i+1$, we should exchange pebbles on $v_{\ell+1} v_{\ell+2}$, $v_{\ell+3} v_{\ell+4}, \ldots$ in $S_{i}$, which are $p_{i_{\ell+1}} p_{i_{\ell+2}}, p_{i_{\ell+3}} p_{i_{\ell+4}}, \ldots$ So, from $T_{i-1}$ to $T_{i}$, we perform the exchange of these pairs of pebbles $p_{(i-1)_{\ell-2}} p_{(i-1)_{\ell-1}}, p_{(i-1)_{\ell-4}} p_{(i-1)_{\ell-3}}, \ldots$, and $p_{i_{\ell+1}} p_{i_{\ell+2}}, p_{i_{\ell+3}} p_{i_{\ell+4}}, \ldots$. This is corresponding to the process of routing the pebbles $p_{2}, \ldots, p_{n-1}$.

Since it takes at most $n-2$ steps to route any permutation on $P_{n-2}$, we have $k-1 \leq n-2$. Hence routing $\pi$ on $P_{n}$ takes at most $k \leq n-1$ steps. $\quad$ Q

Since $P_{n}$ is a spanning subgraph of $C_{n}$, we know $r t\left(C_{n}\right) \leq r t\left(P_{n}\right)$. Using the extra edge in $C_{n}$ not in $P_{n}$, we are ready to prove our second theorem. 
Proof of Theorem 1.2. The lower bound is proved by Lemma 2.1. It suffices to prove

$$
r t\left(C_{n}\right) \leq n-1 .
$$

Given a permutation $\pi$, if $p_{1}$ is on $v_{i}$ and $p_{n}$ is on $v_{j}$ with $i<j$, then we delete the edge $e_{n}$ to get the path $v_{1} v_{2} \ldots v_{n}$. According to Lemma 3.1, we can route $\pi$ in $n-1$ steps. If not, let us cut another edge $e_{k}=v_{k} v_{k+1}$ and observe the pebbles $p_{k}$ and $p_{k+1}$. If on the path $v_{k+1} v_{k+2} \ldots v_{n} v_{1} \ldots v_{k}$, pebble $p_{k}$ is on the right side of $p_{k+1}$, relabel the indices of pebbles and vertices by applying the permutation $\sigma: i \rightarrow i-k \bmod n$. Let $q_{i}=p_{\sigma(i)}$ and $u_{i}=v_{\sigma(i)}$. Apply the odd-even transposition on $q_{i}$ 's and $u_{1} \ldots u_{n}$; we can route the pebbles in $n-1$ steps, but this is exactly the same as routing $\pi$. Thus, the only possible case that we might need $n$ steps to route $\pi$ is no matter what edge we cut to produce a path $v_{k+1} v_{k+2} \ldots v_{n} v_{1} \ldots v_{k}, p_{k}$ always appears on the left side of $p_{k+1}$.

Now assume that at the beginning each $p_{k}$ is on $v_{i_{k}}$. Define $d_{k}$ to be the length of the directed path $v_{i_{k}} v_{i_{k}+1} \ldots v_{k}$ on $C_{n}$. By the previous conclusion, $d_{k} \geq d_{k+1}$ for all $k=1, \ldots n-1$, and $d_{n} \geq d_{1}$. Hence, $d_{1}=d_{2}=\ldots=d_{n}=d$ for some $1 \leq d \leq n-1$. This tells us that $\pi$ is sending $i$ to $d+i \bmod n$ and hence pebble $p_{d+i}$ is on $v_{i}$; the indices are subject to modulo $n$. Therefore, the labeling, induced by $\pi$, of the pebbles on $v_{1} v_{2} \ldots v_{n}$ in order is $p_{d+1} p_{d+2} \ldots p_{n} p_{1} \ldots p_{d}$. Let us call such kind of permutation a $d$-rotation.

If we use the odd-even transposition to route $\pi$ starting from swapping the pebbles on odd edges, then we cannot do any swap whenever $n-d$ is even. Let us modify the odd-even transposition in the following way in order to reduce one step.

For a $d$-rotation $\pi: i \rightarrow d+i \bmod n$ for $1 \leq i \leq n$, we always start the odd-even transposition on edges of the same parity as $n-d$. Thus, the first step is to swap $p_{n}$ and $p_{1}$ on the edge $v_{n-d} v_{n-d+1}$. After the first step, we swap pebbles on odd edges and even edges alternatively.

We show that the above modified odd-even transposition can route $\pi$ in $n-1$ steps.

For $n=3$, the initial distributions of pebbles are $p_{1} p_{2} p_{3}, p_{2} p_{3} p_{1}$, or $p_{3} p_{1} p_{2}$ if $\pi$ is a $d$-rotation. One can easily verify that every pebble can reach its destination in two steps by using the modified odd-even transposition.

Suppose we now need $k$ steps to route a rotation on $P_{n}$. Let $S_{0}, \ldots, S_{k}$ be the lists of pebbles on $v_{1}, v_{2}, \ldots, v_{n}$ as we defined in the proof of Lemma 3.1 after we applied the modified odd-even transposition on $\pi$. Again, if $p_{1}$ is on $v_{\ell}$ after step $i$, then concatenate the pebbles $p_{(i-1)_{1}} \cdots p_{(i-1)_{\ell-1}}$ to $p_{i_{\ell+1}} \cdots p_{i_{n}}$, forming a new list $T_{i-1}$. The new lists $T_{0}, \ldots, T_{k-1}$ show the process of routing a rotation on $P_{n-1}$.

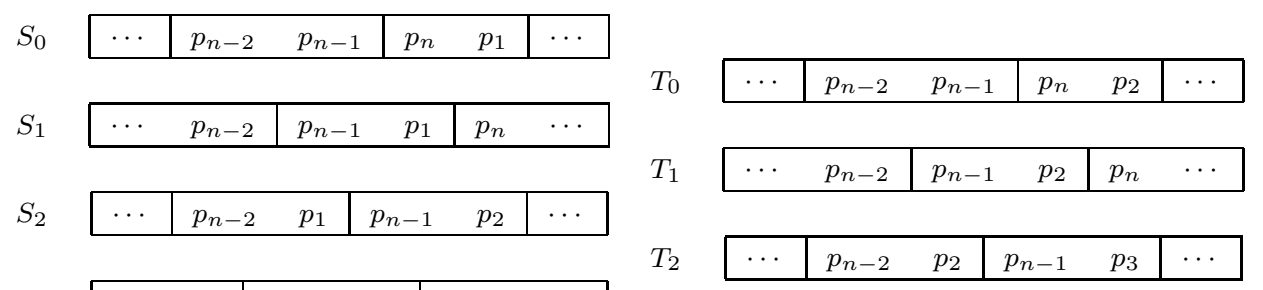

By induction hypothesis, we can show that $k-1 \leq n-2$. Therefore, $k \leq n-1$. 
Proof of Theorem 1.3. Denote the two node sets of $K_{s, t}$ by $X$ and $Y$ such that $|X|=s$ and $|Y|=t$. Given a permutation $\pi$, let $A$ be the set of pebbles in $X$ whose destinations are in $Y$ and $B$ be the set of pebbles in $Y$ whose destinations are in $X$. We always have $|A|=|B|$. If both $A$ and $B$ are empty, do nothing. Otherwise, take one step to swap pebbles in $A$ and $B$ via a perfect matching between them. After this step, all pebbles in $X$ will stay in $X$ and all pebbles in $Y$ will stay in $Y$.

Now route the pebbles in $X$ first. The pebbles in $X$ induce a permutation $\pi^{\prime}$ on $X$. Let $C_{1} \circ C_{2} \circ \cdots \circ C_{m}$ be the disjoint cycle decomposition of $\pi^{\prime}$. When $C_{1} \circ C_{2} \circ \cdots \circ C_{m}$ is chosen, we pose a method to route the pebbles in $X$ in $d+1$ steps. The number $d$ will be determined later $\left(d=\left\lceil\frac{3 s}{2 t}\right\rceil\right)$. In each step of routing we swap some pebbles in $X$ with some pebbles in $Y$. We record the transpositions/swaps of each step in an array as shown below. In this array, $y_{1}, \ldots, y_{t}$ are the $t$ vertices of $Y ; x_{i, j}$ will be assigned a vertex in $X$. If a vertex $x$ in $X$ is assigned to $x_{i, j}$, it means that in step $j$ we swap the pebbles on $x$ and $y_{i}$. Note that we should avoid assigning $x$ to both $x_{i_{1}, j}$ and $x_{i_{2}, j}$ for $i_{1} \neq i_{2}$, since we cannot swap the pebble on $x$ with the pebbles on $y_{i_{1}}$ and $y_{i_{2}}$ simultaneously at step $j$.

\begin{tabular}{|c|c|c|c|c|}
\hline Step 1 & Step 2 & $\cdots$ & Step $d$ & Step $d+1$ \\
\hline$\left(x_{1,1} y_{1}\right)$ & $\left(x_{1,2} y_{1}\right)$ & $\cdots$ & $\left(x_{1, d} y_{1}\right)$ & $\left(x_{1, d+1} y_{1}\right)$ \\
$\left(x_{2,1} y_{2}\right)$ & $\left(x_{2,2} y_{2}\right)$ & $\cdots$ & $\left(x_{2, d} y_{2}\right)$ & $\left(x_{2, d+1} y_{2}\right)$ \\
& & & & \\
$\left(x_{t, 1} y_{t}\right)$ & $\left(x_{t, 2} y_{t}\right)$ & $\cdots$ & $\left(x_{t, d} y_{t}\right)$ & $\left(x_{t, d+1} y_{t}\right)$ \\
\hline
\end{tabular}

We arrange the entries of cycles $C_{1}, \ldots, C_{m}$ into the entries $x_{1,1}, x_{1,2}, x_{1,3} \ldots$, accordingly so that we can route the cycles by doing the indicated transpositions in each step. Start the procedure by the first row. For a cycle $C=\left(a_{1} a_{2} \cdots a_{n}\right)$, if $x_{i, j}, j \leq d$, is not assigned yet, then we assign $a_{1}$ to $x_{i, j}, a_{2}$ to $x_{i, j+1}$, and so on. If $a_{n}$ is assigned to $x_{i, m}, m \leq d$, then we assign $a_{1}$ to $x_{i, m+1}$ again. If some $a_{k}, k<n$, is assigned to $x_{i, d}$, then we skip $x_{i, d+1}$ and go to the $(i+1)$ th row. In addition, we have to assign $a_{k}$ to $x_{i+1,1}$ again, namely, both $x_{i, d}$ and $x_{i+1,1}$ are $a_{k}$.

When $|C| \equiv 0 \bmod (d-1)$, using the above method leads to the following problem. The first $a_{1}$ is assigned to $x_{i_{1}, j}$ and the other $a_{1}$ is in another row but the same column $x_{i_{2}, j}, i_{1} \leq i_{2}$. This is not allowed since the transpositions in the same column must be disjoint. For example, for $d=6$ and $C=\left(a_{1} a_{2} \ldots a_{10}\right)$, we have $|C|=10$, which is divisible by $d-1=5$. (See the following array.)

\begin{tabular}{|l|l|l|l|l|l|l|}
\hline Step 1 & Step 2 & Step 3 & Step 4 & Step 5 & Step 6 & Step 7 \\
\hline & & & $\left(\mathbf{a}_{\mathbf{1}} \mathbf{y}_{\mathbf{1}}\right)$ & $\left(a_{2} y_{1}\right)$ & $\left(a_{3} y_{1}\right)$ & \\
$\left(a_{3} y_{2}\right)$ & $\left(a_{4} y_{2}\right)$ & $\left(a_{5} y_{2}\right)$ & $\left(a_{6} y_{2}\right)$ & $\left(a_{7} y_{2}\right)$ & $\left(a_{8} y_{2}\right)$ & \\
$\left(a_{8} y_{3}\right)$ & $\left(a_{9} y_{3}\right)$ & $\left(a_{10} y_{3}\right)$ & $\left(\mathbf{a}_{\mathbf{1}} \mathbf{y}_{\mathbf{3}}\right)$ & & & \\
\hline
\end{tabular}

If this happens, we must have assigned $a_{d-j+1}$ to $x_{i, d}$ and $x_{i+1,1}$ beforehand. We now solve this problem using $x_{i, d+1}$. We assign $a_{d-j+1}$ to $x_{i, d}$, then $a_{d-j+2}$ to $x_{i, d+1}$ and $x_{i+1,1}$. For the remaining $a_{i}$ 's, we still follow previous rules. Here is the solution of the previous example.

\begin{tabular}{|l|l|l|l|l|l|l|}
\hline Step 1 & Step 2 & Step 3 & Step 4 & Step 5 & Step 6 & Step 7 \\
\hline & & & $\left(a_{1} y_{1}\right)$ & $\left(a_{2} y_{1}\right)$ & $\left(a_{3} y_{1}\right)$ & $\left(a_{4} y_{1}\right)$ \\
$\left(a_{4} y_{2}\right)$ & $\left(a_{5} y_{2}\right)$ & $\left(a_{6} y_{2}\right)$ & $\left(a_{7} y_{2}\right)$ & $\left(a_{8} y_{2}\right)$ & $\left(a_{9} y_{2}\right)$ & \\
$\left(a_{9} y_{3}\right)$ & $\left(a_{10} y_{3}\right)$ & $\left(a_{1} y_{3}\right)$ & & & & \\
\hline
\end{tabular}

Since all cycles are routed by this procedure, permutation $\pi^{\prime}$ is routed. 
Let us determine how small $d$ could be. Each entry $a_{i}$ in the cycle $\left(a_{1} a_{2} \cdots a_{n}\right)$ appears either once or twice in the array. The entry $a_{1}$ must appear twice. If an entry $a_{k}$ (not $a_{1}$ and $a_{n}$ ) in a cycle is assigned to $x_{i, d}$, then it is assigned to $x_{i+1,1}$ as well. The number of this kind of entry cannot exceed $t$. Hence we assign at most $\left|C_{1}\right|+\cdots+\left|C_{m}\right|+m+t$ entries to $t(d+1)$ entries $x_{i, j}$ 's. Furthermore, $\left|C_{1}\right|+\cdots+$ $\left|C_{m}\right|+m+t \leq|X|+\frac{|X|}{2}+t \leq \frac{3 s}{2}+t$. So we have the inequality,

$$
t(d+1) \geq \frac{3 s}{2}+t .
$$

This means that when $d=\left\lceil\frac{3 s}{2 t}\right\rceil$ the above procedure works. Hence the number of steps needed to route $\pi^{\prime}$ is no more than $\left\lceil\frac{3 s}{2 t}\right\rceil+1$. Finally, we route the pebbles on $Y$, which have not been moved to their destinations. Select any $t$ routed pebbles in $X$ together with the pebbles in $Y$ which induce a permutation on $K_{t, t}$. It can be routed in four steps (see [1]). Therefore $r t\left(K_{s, t}\right) \leq 1+\left\lceil\frac{3 s}{2 t}\right\rceil+5 \leq\left\lfloor\frac{3 s}{2 t}\right\rfloor+7$.

Consider the permutation $\sigma=\left(x_{1} x_{2}\right)\left(x_{3} x_{4}\right) \cdots\left(x_{2\left\lfloor\frac{s}{2}\right\rfloor-1} x_{2\left\lfloor\frac{s}{2}\right\rfloor}\right)$. For each $\left(x_{2 i-1} x_{2 i}\right)$, we need at least three transpositions to route it. (We need the pebbles on $x_{2 i-1}$ and $x_{2 i}$ leaving their original places and also entering their destinations. One can only save one move by moving the pebble $p_{x_{2 i}}$ on $x_{2 i-1}$ out and moving the pebble $p_{x_{2 i-1}}$ into $x_{2 i-1}$ at the same time.) Therefore, it takes at least $\left\lceil\frac{3\left\lfloor\frac{s}{2}\right\rfloor}{t}\right\rceil$ steps. Since $1+\left\lceil\frac{3 s-3}{2 t}\right\rceil \geq 1+\frac{3 s-3}{2 t} \geq \frac{3 s}{2 t}$, we have $1+\left\lceil\frac{3 s-3}{2 t}\right\rceil \geq\left\lceil\frac{3 s}{2 t}\right\rceil$. Thus, $\left\lceil\frac{3\left\lfloor\frac{s}{2}\right\rfloor}{t}\right\rceil \geq\left\lceil\frac{3 s-3}{2 t}\right\rceil \geq\left\lceil\frac{3 s}{2 t}\right\rceil-1 \geq\left\lfloor\frac{3 s}{2 t}\right\rfloor-1$. This gives the lower bound for $r t\left(K_{s, t}\right)$.

4. Fractional routing numbers. We can relax the routing number $\operatorname{rt}(G)$ to the fractional routing number $r t^{\prime}(G)$ as follows. We assume that all pebbles have mass 1 and can be split into smaller pieces during the routing process. A piece from pebble $p_{i}$ is said to have a type $p_{i}$. After reaching its destination, all pieces of type $p_{i}$ can be assembled into the pebble $p_{i}$. The pieces can be exchanged through a fractional matching at one step. A fractional matching is a mapping

$$
f: E(G) \rightarrow[0,1]
$$

satisfying for any $v, \sum_{u \in \Gamma(v)} f(u v) \leq 1$. Recall that $\Gamma(v)$ is the set of neighbors of $v$ in $G$. For each edge $u v$, pieces of total mass $f(u v)$ at $u$ can be exchanged with pieces of the same total mass $f(u v)$ at $v$. Given a permutation $\pi$, the pebble on $v_{i}$ will be labeled as $p_{j}$ if $\pi(i)=j$. The minimum number of steps to route each pebble $p_{i}$ to $v_{i}$ is denoted by $r t^{\prime}(G, \pi)$. Finally, we define $r t^{\prime}(G)$, the fractional routing number of $G$, by

$$
r t^{\prime}(G)=\max _{\pi} r t^{\prime}(G, \pi) .
$$

Since every matching is a fractional matching, we have

$$
r t^{\prime}(G) \leq r t(G) .
$$

Using this relation and the fact that $r t\left(K_{n}\right)=2$, we can get $r t^{\prime}\left(K_{n}\right)=2$ for $n \geq 3$.

Many lower bounds for routing numbers are actually lower bounds for the fractional routing numbers. For example,

$$
r t^{\prime}(G) \geq D(G) .
$$


Lemma 4.1. Suppose $C$ is a vertex-cut of a connected graph $G$, and the largest component after removing $C$ has at most $\frac{n-|C|}{2}$ vertices. Then,

$$
r t^{\prime}(G) \geq \frac{n}{|C|} .
$$

Alon, Chung, and Graham [1] proved

$$
r t(G) \geq \frac{2}{|C|} \min \{|A|,|B|\},
$$

where $A \cup B$ is a partition of $V(G) \backslash C$ so that there is no edge between $A$ and $B$. The right hand side of inequality (4.3) reaches the maximum when $|A|=|B|=\frac{n-|C|}{2}$. In this case, inequality (4.3) gives $r t(G) \geq \frac{n}{|C|}-1$. Lemma 4.1 gives a slightly better lower bound $r t^{\prime}(G) \geq \frac{n}{|C|}$.

Lemma 4.2. Let $\cup_{i=1}^{r} S_{i}$ be a partition of a set $S(|S| \geq 2)$ satisfying

$$
\left|S_{i}\right| \leq \frac{|S|}{2} \quad \text { for all } i
$$

Then there is a permutation $\pi$ on $S$ so that for any $v \in S, \pi(v)$ and $v$ are in different blocks.

Proof. Relabel the vertices if necessary. Without loss of generality, we can assume that the sets $S_{i}$ consist of consecutively numbered vertices (e.g., $S_{1}=\left\{1,2, \ldots, i_{1}\right\}$, $S_{2}=\left\{i_{1}+1, i_{1}+2, \ldots, i_{2}\right\}, \ldots$, etc.) Consider the permutation

$$
\pi: i \rightarrow i+\left\lfloor\frac{|S|}{2}\right\rfloor \bmod |S| .
$$

We claim $i$ and $\pi(i)$ are not in the same block. If not, there is a block $S_{j}$ containing both $i$ and $\pi(i)$. Then $S_{j}$ contains the interval $[i, \pi(i)]$ if $i<\pi(i)$, or the interval $[\pi(i), i]$ if $i>\pi(i)$. In either case, it contradicts $\left|S_{i}\right| \leq \frac{|S|}{2}$.

Proof of Lemma 4.1. Suppose that the connected components of $G \backslash C$ are $C_{1}, C_{2}, \ldots, C_{r}$. By Lemma 4.2, there exists a permutation $\sigma$ on $C_{1} \cup C_{2} \cup \cdots \cup C_{r}$ so that for any vertex $v, \sigma(v)$ and $v$ are always in different components. Extend this permutation $\sigma$ over $V(G)$ so that the vertices in $C$ are fixed points. Any path from $v$ to $\sigma(v)$ must go through some vertex in $C$. The total mass of such pieces to get in and get out of $C$ is at least $2(n-|C|)$. For any routing process of $\sigma$, at each step the total mass of such pieces that can get in and get out of $C$ is at most $2|C|$. We also observe that at the first step and the last step, the amount of such pieces can get in and get out of $C$ is at most $|C|$. Thus the number of steps is at least

$$
\frac{2(n-|C|)}{2|C|}+1=\frac{n}{|C|} .
$$

Theorem 4.3. For any tree $T$ on $n \geq 3$ vertices, we have

$$
r t^{\prime}(T) \geq n .
$$

Proof. For a vertex $u$, let $f(u)$ be the maximum size of connected components after deleting $u$ from $T$. Let $v$ be a vertex so that $f(v)$ reaches the minimum value. There are two cases. 
Case 1. $f(v) \leq \frac{n-1}{2}$. Applying inequality (4.2) with $C=\{v\}$, we have $r t^{\prime}(T) \geq n$.

Case 2. $f(v) \geq \frac{n}{2}$. Let $u$ be the neighbor of $v$ so that the component containing $u$ is of size $f(v)$. Among the components of $T \backslash\{u\}$, the one containing $v$ has at most $\frac{n}{2}$ vertices. All other components have at most $f(v)-1$ vertices. Since $f(u) \geq f(v)$, we have $f(u) \leq \frac{n}{2}$. In particular, we have $f(v)=f(u)=\frac{n}{2}$, and $n$ is even. So the edge $u v$ cuts the tree $T$ into two subtrees (denoted by $T_{u}$ and $T_{v}$ ) of an equal size. Let $\pi$ be a permutation of $V(T)$ that maps any vertex of $T_{u} \backslash\{u\}$ to a vertex of $T_{v} \backslash\{v\}$, $\pi(u)=v$, and $\pi(v)=u$.

Let $k$ be the number of steps in the routing process. For $1 \leq i \leq k$, let $a_{i}$ be the mass of pieces of types in $T_{v}$, which first goes through the edge $u v$ at the $i$ th step. At $(i+1)$ th step, at least $a_{i}$ mass at vertex $u$ was from vertex $v$. By the definition of $a_{i+1}$, we have

$$
a_{i+1} \leq 1-a_{i}
$$

for any $1 \leq i \leq k-1$. Note the first step and the last step can only route the piece of type $v$. We also have $a_{1}+a_{k} \leq 1$.

$$
\begin{aligned}
\frac{n}{2} & \leq \sum_{i=1}^{k} a_{i} \\
& =\frac{1}{2}\left[\left(a_{1}+a_{k}\right)+\sum_{i=1}^{k-1}\left(a_{i}+a_{i+1}\right)\right] \\
& \leq \frac{1}{2} k .
\end{aligned}
$$

This implies $k \geq n$.

COROLlary 4.4. For any tree $T$ on $n \geq 3$ vertices, we have

$$
r t(T) \geq n .
$$

For $n \geq 3$, we have $r t^{\prime}\left(T_{n}\right)=n$. For the star $S_{n}$, it is known that $r t\left(S_{n}\right)=\left\lfloor\frac{3(n-1)}{2}\right\rfloor$. Here we show $r t^{\prime}\left(S_{n}\right)$ is much smaller than $r t\left(S_{n}\right)$.

TheOREM 4.5. For the star $S_{n}$ on $n \geq 2$ vertices, we have

$$
r t^{\prime}\left(S_{n}\right)=n .
$$

Proof. Let $c$ be the center of the star $S_{n}$ and $\pi$ be any permutation on $V\left(S_{n}\right)$. $\pi$ can be written as the product of $r$ disjoint circles $C_{1} \circ C_{2} \circ \cdots C_{r}$. We also assume the center $c$ is in $C_{1}$. Let $\sigma=C_{2} \circ \cdots C_{r}$. We will route pebbles in $C_{1}$ first.

Claim (a). The cycle $C_{1}$ can be routed in $\left|C_{1}\right|-1$ steps.

Claim (a) is trivial for $\left|C_{1}\right|=1$. Now we assume $\left|C_{1}\right|=s+1$. Relabeling the vertices if needed, we assume $C_{1}=(c, 1,2, \ldots, s)$. First swap $p_{c}$ and $p_{1}$, then $p_{1}$ and $p_{2}$, and so on. The last step is to swap $p_{s-1}$ and $p_{s}$. Claim (a) is proved.

Claim (b). The permutation $\sigma$ can be routed in $n-\left|C_{1}\right|+1$ steps.

The vertices outside $C_{1}$ are labeled by $s+1, s+2, \ldots, n-1$. The pebbles on these vertices are divided into $n-s-1$ pieces of equal mass $\frac{1}{n-s-1}$. At the first step, pick one piece at each vertex and swap it with a piece at the center. Now the center contains exactly $n-s-1$ pieces of all different types (other than type $c$ ). Each vertex outside $C_{1}$ has exactly one piece of type $c$. At the subsequent $n-s-2$ steps, for $s+1 \leq j \leq n-1$, pick one piece of type $j$ at the center and swap it with a 
piece of type $\sigma(j)$ at vertex $j$. The center always contains exactly $n-s-1$ pieces of all different types (other than type $c$ ). At the last step, pick a piece of type $j$ at the center and swap it with the piece of type $c$ at vertex $j$. The number of steps is $n-s=n-\left|C_{1}\right|+1$. The proof of Claim (b) is finished.

Thus for any permutation $\pi$, we have

$$
r t^{\prime}\left(S_{n}, \pi\right) \leq\left|C_{1}\right|-1+n-\left|C_{1}\right|+1=n .
$$

The upper bound $r t^{\prime}\left(S_{n}\right) \leq n$ is proved. The lower bound $r t^{\prime}\left(S_{n}\right) \geq n$ is obtained by Theorem 4.3.

One can prove the following lemma analogous to Lemma 2.1. Here we omit its proof.

Lemma 4.6. For the cycle $C_{n}$ on $n$ vertices, we have

$$
r t^{\prime}\left(C_{n}\right) \geq n-1 \text {. }
$$

Thus for the cycles, we have the following theorem.

Theorem 4.7. For the cycle $C_{n}$ on $n \geq 3$ vertices, we have

$$
r t^{\prime}\left(C_{n}\right)=n-1 \text {. }
$$

Goddard (see [1]) showed $r t\left(K_{n, n}\right)=4$. Here we determine $r t^{\prime}\left(K_{n, n}\right)$.

Theorem 4.8. For $n \geq 2$, we have

$$
r t^{\prime}\left(K_{n, n}\right)=3 .
$$

Proof. Denote the two vertex sets of $K_{n, n}$ by $X$ and $Y$ such that $|X|=|Y|=n$. Given a permutation $\pi$, if there are some pebbles in $X$ whose destinations are in $Y$, then there will be the same number of pebbles in $Y$ whose destinations are in $X$. The first step is to swap these pebbles so that all pebbles in $X$ have destinations in $X$ and also all pebbles in $Y$ will stay in $Y$. Then we divide each pebble into $n$ pieces of mass $\frac{1}{n}$. The fractional matching we used here is $f(x y)=\frac{1}{n}$ for any edge $x y$. The second step is to swap pairs of pieces through all edges. After this step, each vertex on $X$ has $n$ different pieces from $Y$, while each vertex on $Y$ has $n$ different pieces from $X$. At the last step, for each edge $x_{i} y_{j}$, swap the pair of pieces which belong to their destinations. Thus,

$$
r t^{\prime}\left(K_{3,3}\right) \leq 3
$$

The lower bound comes from a special permutation $\sigma$. Let $x_{1}, x_{2} \in X$ and $y_{1}, y_{2} \in$ $Y$. The induced graph on these four vertices is $K_{2,2}=C_{4}$. Let $\sigma$ be the permutation of a rotation on these four vertices. Namely, for any $v \in X \cup Y$,

$$
\sigma(v)= \begin{cases}y_{1} & \text { if } v=x_{1} \\ x_{2} & \text { if } v=y_{1} \\ y_{2} & \text { if } v=x_{2} \\ x_{1} & \text { if } v=y_{2} \\ v & \text { otherwise }\end{cases}
$$

We would like to show $r t^{\prime}\left(K_{n, n}, \sigma\right) \geq 3$. If a routing process routes a piece of type $x_{1}$ to a vertex $u\left(u \neq x_{1}, x_{2}, y_{1}, y_{2}\right)$, then any walk from $x_{1}$ to $y_{1}$ passing through $u$ needs at least three steps since $K_{n, n}$ is a bipartite graph. The same argument applies to 
the pieces of type $x_{2}, y_{1}, y_{2}$. Thus, we can assume that no piece of type $x_{1}, y_{1}, x_{2}, y_{2}$ is routed out of these four vertices. When we restrict to $x_{1}, y_{1}, x_{2}, y_{2}$, it induces a routing process on $C_{4}$. Since $r t^{\prime}\left(C_{4},\left.\sigma\right|_{\left\{x_{1}, y_{1}, x_{2}, y_{2}\right\}}\right)=3$, we need at least three steps. The proof is finished.

Lemma 2.2 can also be generalized to the following lemma.

LEMmA 4.9. Let $G$ be an m-routing graph, then

$$
r t^{\prime}\left(C_{n} \square G\right) \geq n+m-1 .
$$

In particular, we have

$$
r t^{\prime}\left(Q_{n}\right) \geq n+1
$$

\section{Concluding remarks.}

- The routing number can be computed as follows. For an edge $e=v_{i} v_{j} \in E(G)$, let $P_{e}=(i j)$ be the swapping permutation of $i$ and $j$. For a matching $M$, the move of swapping through the set of edges in $M$ can be written as a permutation

$$
P_{M}=\prod_{e \in M} P_{e}
$$

Consider an auxiliary Cayley graph $H=\left(\right.$ Perm $\left._{n}, K\right)$, where Perm $_{n}$ is the permutation group and $K=\left\{P_{M}\right.$ : for any matching $\left.M\right\}$. For any permutations $\pi$ and $\sigma$, the graph distance in $H$ from $\sigma$ to $\pi$ in $G$ is exactly $r t\left(G, \sigma^{-1} \pi\right)$. In particular, $r t(G)$ is the diameter of the graph $H$. So the value of $r t(G)$ does not depend on the labeling of vertices. This also suggests a simple algorithm for computing the $r t(G)$. Namely, construct the auxiliary graph $H$ and apply the breadth-first-search algorithm to $H$. The worst-case running time of this algorithm is $O(|V(H)|+|E(H)|)=O\left((n !)^{2}\right)$. We implemented this algorithm and computed the routing number of any connected graph up to 8 vertices in this way. We found out that $r t\left(Q_{3}\right)=4$. This approach fails miserably when we try to compute $r t\left(Q_{4}\right)$.

Question: Is there an efficient algorithm to compute $\operatorname{rt}(G, \pi)$ ?

- In the proof of Theorem 1.2, we prove that $\operatorname{rt}\left(C_{n}, \pi\right)=n-1$ if $\pi$ is the rotation $(123 \cdots n)$ or its inverse.

Conjecture 1. For $n \geq 5$, if $r t\left(C_{n}, \pi\right)=n-1$, then $\pi$ is the rotation $(123 \cdots n)$ or its inverse. (Here we label the vertices of $C_{n}$ in the standard way.)

The outputs of our program suggest this conjecture holds for $n=5,6,7,8$. For $n=4$, there are four permutations $\pi=(1234),(4321),(13)$, and (24) giving $r t\left(C_{4}, \pi\right)=3$. We didn't observe a similar pattern for the path $P_{n}$.

- Many lower bounds for routing numbers are actually the lower bounds for the corresponding fractional routing numbers. This observation motivates us to consider the fractional routing numbers. We proved $r t^{\prime}(T) \geq n$, and the inequality holds for the paths and the stars. In many extremal situations, paths and stars are usually in the opposite direction. We conclude our paper with the following conjecture.

Conjecture 2. For any tree $T$ on $n \geq 3$ vertices, $r t^{\prime}(T)=n+O(1)$. Maybe even $r t^{\prime}(T)=n$ holds. 
Acknowledgment. We would like to thank the anonymous referees for their valuable comments. The current elegant proof of Lemma 4.2 is due to one of the anonymous referees.

\section{REFERENCES}

[1] N. Alon, F. R. K. Chung, and R. L. Graham, Routing permutations on graphs via matchings, SIAM J. Discrete Math., 7 (1994), pp. 513-530.

[2] M. Baumslag and F. Annexstein, A unified framework for off-line permutation routing in parallel networks, Math. Systems Theory, 24 (1991), pp. 233-251.

[3] V. E. BenĚs, Mathematical Theory of Connecting Networks, Academic Press, New York, 1965.

[4] F. K. Chung, Spectral Graph Theory, AMS Publications, Providence, RI, 1997.

[5] D. E. Knuth, The Art of Computer Programming, Vol. 3, Addison-Wesley, Reading, MA, 1973, p. 241.

[6] M. RAmRAS, Routing permutations on a graph, Networks, 23 (1993), pp. 391-398.

[7] L. Valiant, A scheme for fast parallel communication, SIAM J. Comput., 11 (1982), pp. 350361.

[8] L. ZhAnG, Optimal bounds for matching routing on trees, SIAM J. Discrete Math., 12 (1999), pp. $64-77$. 